Research Article

\title{
A Framework for Nonconforming Mixed Finite Element Method for Elliptic Problems in $\mathbb{R}^{3}$
}

\author{
Gwanghyun Jo' and J. H. Kim $\mathbb{D}^{2}$ \\ ${ }^{1}$ Department of Mathematics, Kunsan National University, Gunsan-si, Jeollabuk-do, Republic of Korea \\ ${ }^{2}$ Department of Mathematics, Hannam University, Daejeon 306-791, Republic of Korea \\ Correspondence should be addressed to J. H. Kim; kimjh@hnu.kr
}

Received 11 September 2019; Accepted 11 March 2020; Published 9 April 2020

Academic Editor: Dogan Kaya

Copyright (c) 2020 Gwanghyun Jo and J. H. Kim. This is an open access article distributed under the Creative Commons Attribution License, which permits unrestricted use, distribution, and reproduction in any medium, provided the original work is properly cited.

\begin{abstract}
In this paper, we suggest a new patch condition for nonconforming mixed finite elements (MFEs) on parallelepiped and provide a framework for the convergence. Also, we introduce a new family of nonconforming MFE space satisfying the new patch condition. The numerical experiments show that the new MFE shows optimal order convergence in $H$ (div) and $L^{2}$-norm for various problems with discontinuous coefficient case.
\end{abstract}

\section{Introduction}

The finite element method has achieved great success in many fields, and it has become a powerful tool for solving partial differential equations [1-3]. The main idea of the finite element method is using a finite dimensional space to approximate the exact solution on the given space according to a certain kind of variational principle. In the finite element discretizations, a basic distinction can be made between conforming and nonconforming methods. When the finite element space is a subspace of the solution space, the method is called conforming. In this case, the error between the true solution and the finite element method (FEM) solution is bounded by the distance between the FEM space and the given space by Céa's lemma. Meanwhile, the nonconforming finite element space is not contained in the space the exact solution lives in. Hence, extra error committed by the nonconformity has to be estimated.

There are some situations in which finite element methods for the primary variable do not yield satisfactory results, such as elliptic problem with large jumps in the diffusion coefficient. Sometimes, other quantities such as Darcy velocity along with pressure of the flow in the porous media become variables of main interest. In this case, the mixed finite element method (MFEM) is preferred. Many mixed finite element methods have been developed since it was first suggested in the late 1970s [4-6]. The idea of the mixed methods is to introduce the velocity as a new variable and change the given equation into a system of equations. By discretizing this system, we can compute two variables, velocity and pressure, simultaneously and expect a more accurate velocity. MFEM has been used in many applications such as porous media problem $[7,8]$ and chemical engineering [9].

The nonconforming approaches have been widely studied for Lagrangian finite elements. So far, all the wellknown mixed finite element (MFE) space are conforming in the sense that the space is contained in $H(\operatorname{div}, \Omega)$, defined as the space of all vector functions whose divergence belongs to $L^{2}(\Omega)$. It is natural to ask whether there exists a nonconforming counterpart of the MFE space. Hiptmair [10] has investigated some conditions for the nonconforming MFE space. But, under the conditions suggested there, they only show suboptimal convergence. Meanwhile, a family of high-order nonconforming MFE space was introduced in [11] a few years ago, and numerical examples show the optimal order of convergence. However, there is no analysis.

In this paper, we suggest a new condition under which the nonconforming MFEM may have optimal convergence. In addition, we introduce another family of the 
nonconforming MFE space which satisfies this condition. To the author's best knowledge, nonconforming element having optimal order has not been suggested by others.

The organization of this paper is as follows: in the next section, we present the model problem. In Section 3, we introduce nonconforming mixed finite element spaces on parallelepiped in $\mathbb{R}^{3}$ together with a new patch condition. A framework for the convergence is given in Section 4 . We give numerical experiments in Section 5.

\section{Model Problem}

Given $\Omega \subset \mathbb{R}^{3}$, a simply connected bounded Lipschitz polyhedral domain with connected boundary $\partial \Omega$, we consider the following second-order elliptic problem:

$$
\begin{cases}-\operatorname{div}(\kappa \nabla p)=f, & \text { in } \Omega, \\ p=0, & \text { on } \partial \Omega,\end{cases}
$$

where $\kappa \in L^{\infty}(\Omega)$ is assumed to be uniformly positive definite and bounded. And $f$ is a given function in $L^{2}(\Omega)$. To write the given equation into a mixed system, we use Darcy's law, $\mathbf{u}=-\kappa \nabla p$. Then, we can rewrite problem (1) in the following mixed form:

$$
\begin{cases}\mathbf{u}+\kappa \nabla p=0, & \text { in } \Omega, \\ \operatorname{div} \mathbf{u}=f, & \text { in } \Omega, \\ p=0, & \text { on } \partial \Omega .\end{cases}
$$

Denote by $\mathbf{V}=H(\operatorname{div}, \Omega)=\left\{\mathbf{v} \in\left(L^{2}(\Omega)\right)^{3}: \operatorname{div} \mathbf{v} \in L^{2}(\Omega)\right\}$ and $W=L^{2}(\Omega)$ the usual Sobolev spaces with obvious norms. Then, we have the following variational form for (2): find $(\mathbf{u}, p) \in \mathbf{V} \times W$ such that

$$
\begin{aligned}
& \int_{\Omega} \kappa^{-1} \mathbf{u v d} \mathbf{x}-\int_{\Omega} p \operatorname{div} \mathbf{v} \mathrm{d} \mathbf{x}=0, \quad \forall \mathbf{v} \in \mathbf{V}, \\
& \int_{\Omega} \operatorname{div} \mathbf{u} \cdot q \mathrm{~d} \mathbf{x}=\int_{\Omega} f q \mathrm{~d} \mathbf{x}, \quad \forall q \in W .
\end{aligned}
$$

For the convenience of the presentation, we let

$$
\begin{aligned}
& a(\mathbf{u}, \mathbf{v})=\int_{\Omega} \kappa^{-1} \mathbf{u v} \mathrm{d} \mathbf{x}, \\
& b(\mathbf{v}, p)=-\int_{\Omega} p \operatorname{div} \mathbf{v} \mathrm{d} \mathbf{x} .
\end{aligned}
$$

Then, saddle point problems (3) and (4) can be expressed simply as follows: find $(\mathbf{u}, p) \in \mathbf{V} \times W$ such that

$$
\begin{aligned}
a(\mathbf{u}, \mathbf{v})+b(\mathbf{v}, p) & =0, \quad \forall \mathbf{v} \in \mathbf{V}, \\
b(\mathbf{u}, q) & =-(f, q), \quad \forall q \in W,
\end{aligned}
$$

where $(\cdot, \cdot)$ indicates the inner product in $L^{2}(\Omega)$. If familiar inf-sup condition holds, then problem (6) has a unique solution [12].

\section{Nonconforming Mixed Finite Element Spaces}

The main idea of MFEM is solving problem (6) over suitable locally defined finite dimensional spaces. Their construction depends on triangulations $\mathscr{T}_{h}$ of $\Omega$. Let $\Omega=[0,1]^{3}$ and
$\left\{\mathscr{T}_{h}: h>0\right\}$ be a family of partitions of $\Omega$ into parallelepiped $K$ obtained by uniform division having each side length $h$.

For the construction of the nonconforming MFE space $\mathbf{V}_{h}$, we require that

$$
\begin{aligned}
& \text { (1) } \mathbf{V}_{h}=\left\{\mathbf{v} \in\left(L^{2}(\Omega)\right)^{3}|\mathbf{v}|_{K} \in H(\operatorname{div}, K), \quad \forall K \in \mathscr{T}_{h}\right\}, \\
& \text { (2) } \mathbf{V}_{h} \nsubseteq \mathbf{V}=H(\operatorname{div}, \Omega) .
\end{aligned}
$$

That is, a function in the space $\mathbf{V}_{h}$ is locally in $H(\operatorname{div}, K)$ but not in $H(\operatorname{div}, \Omega)$ over the triangulation $\mathscr{T}_{h}$. A lack of continuity of normal components across interelement boundaries gives rise to a nonconforming approximation. But, we still require some local conformity.

For any domain $D$ in $\mathbb{R}^{2}$ or $\mathbb{R}^{3}$, let $P_{\ell}(D)$ be the space of polynomials of total degree $\ell$ and $Q_{\ell, m}(D)$ or $Q_{\ell, m, n}(D)$ be the space of polynomials of degree less or equal to $\ell, m, n$, respectively, in each variable.

3.1. Patch Conditions. First, we recall a well-known type of "patch condition": let $f$ be the common face of two adjacent elements $K_{i}$ and $K_{j}$.

$$
\begin{array}{r}
\text { (H1) } \int_{f} q\left(\left.\mathbf{v}_{h}\right|_{K_{i}} \cdot \mathbf{n}-\left.\mathbf{v}_{h}\right|_{K_{j}} \cdot \mathbf{n}\right) \mathrm{d} A=0, \\
\forall q \in P_{\ell}(f) \text { for some } \ell=1,2, \ldots,
\end{array}
$$

where $\mathbf{n}$ is an outer unit normal vector to each element. For example, Hiptmair used this condition together with the assumption of continuous interpolation and showed a suboptimal error estimate [10]. However, for the 3D case (parallelepiped), we see that such condition is not enough to guarantee the existence of continuous interpolation which is necessary to derive an approximation. With hypothesis $(H 1)$ holding for $q \in P_{\ell}(f)$ is not enough to determine a continuous interpolation over the whole domain $\Omega$. In fact, if such an interpolation exists, then $(H 1)$ holds for $q \in Q_{\ell, \ell}(f)$, which would imply the conformity of the space $\mathbf{V}_{h}$. Thus, to study a nonconforming MFE, we need a stronger patch condition but not strong enough to make the $\mathbf{V}_{h}$ space fully conforming.

So, we suggest new patch conditions $(H 2)$ : for all vertical faces $f_{V}:=\partial K_{i} \cap \partial K_{j}(i \neq j)$ and all $\mathbf{v}_{h} \in \mathbf{V}_{h}$, we have

$$
\left(H 2 \text { (a)) } \int_{f_{V}} q\left(\left.\mathbf{v}_{h}\right|_{K_{i}} \cdot \mathbf{n}-\left.\mathbf{v}_{h}\right|_{K_{j}} \cdot \mathbf{n}\right) \mathrm{d} A=0, \quad \forall q \in Q_{\ell, \ell}\left(f_{V}\right) .\right.
$$
have

For horizontal faces $f_{H}=\partial K_{i} \cap \partial K_{j}$ and all $\mathbf{v}_{h} \in \mathbf{V}_{h}$, we

$$
\begin{gathered}
\left(H 2 \text { (b)) } \int_{f_{H}} q\left(\left.\mathbf{v}_{h}\right|_{K_{i}} \cdot \mathbf{n}-\left.\mathbf{v}_{h}\right|_{K_{j}} \cdot \mathbf{n}\right) \mathrm{d} A=0,\right. \\
\forall q \in Q_{\ell, \ell}\left(f_{H}\right) \backslash\left\{x^{k} y^{k}\right\} .
\end{gathered}
$$

This means that the moments up to order $\ell$ of the discrete velocity are continuous across horizontal interelement boundaries with respect to $Q_{\ell, \ell}\left(f_{V}\right)$, and the moments 
across vertical interelement boundaries are continuous up to $Q_{\ell, \ell}\left(f_{H}\right) \backslash\left\{x^{k} y^{k}\right\}$ only.

Now, we introduce a new nonconforming MFE. We denote by $Q_{\ell, m, n}^{*}$ the set of all polynomials of $Q_{\ell, m, n}$ except those having the form $x^{\ell} y^{m} z^{p}$ for $p=1, \ldots, n$.

Definition 1. Let $\mathbf{V}_{h}(K)$ be the subspace of

$$
\begin{aligned}
& Q_{k+1, k, k}^{*}(K) \times Q_{k, k+1, k}^{*}(K) \\
& \times\left(Q_{k, k, k+1}^{*}(K) \oplus\left\{x^{k+1} y^{i}, x^{i} y^{k+1}, i=0, \ldots, k-1\right\}\right),
\end{aligned}
$$

where the elements $\left(x^{k+1} y^{k}, 0,0\right)$ and $\left(0, x^{k} y^{k+1}, 0\right)$ are replaced by the single element $\left(x^{k+1} y^{k},-x^{k} y^{k+1}, 0\right)$.

We note that this element is similar to [11], but the number of DOFs is reduced by 2 on each element.

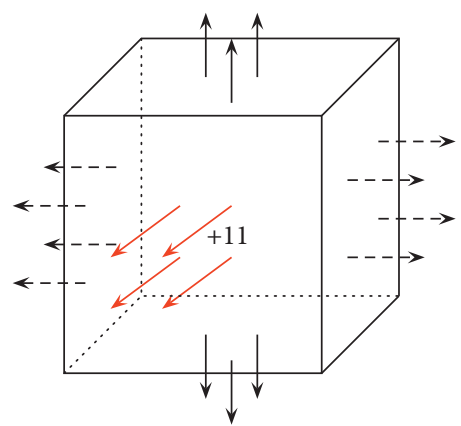

FIgURE 1: DOFs when $k=1,4$ for vertical faces and 3 for horizontal faces.

Then, the dimension of $\mathbf{V}_{h}(K)$ is $2\left\{(k+2)(k+1)^{2}-k\right\}+$ $\left\{(k+1)^{2}(k+2)-(k+1)+2 k\right\}-1=3 k^{3}+12 k^{2}+14 k+4$. For $k=1$, we have $\mathbf{u}=\left(u_{1}, u_{2}, u_{3}\right)$, where

$$
\begin{aligned}
& u_{1}=P_{1}(x, y, z)+a_{1} x y+a_{2} y z+a_{3} z x+a_{4} x y z+a_{5} x^{2}+a_{6} x^{2} z+d x^{2} y, \\
& u_{2}=P_{1}(x, y, z)+b_{1} x y+b_{2} y z+b_{3} z x+b_{4} x y z+b_{5} y^{2}+b_{6} y^{2} z-d x y^{2}, \\
& u_{3}=P_{1}(x, y, z)+c_{1} x y+c_{2} y z+c_{3} z x+c_{4} z^{2}+c_{5} x z^{2}+c_{6} y z^{2}+c_{7} x^{2}+c_{8} y^{2} .
\end{aligned}
$$

This has 33 unknowns in each element, in which 3 less than the RTN space [5] (see Figure 1).

To define the degrees of freedom, we need an auxiliary space. Let $\Psi_{h}(K)$ be the subspace consisting of element type $\phi=\left(\phi_{1}, \phi_{2}, 0\right)$ :

$$
Q_{k-1, k, k}^{*}(K) \times Q_{k, k-1, k}^{*}(K) \times\{0\},
$$

where the elements $\left(x^{k-1} y^{k}, 0,0\right)$ and $\left(0, x^{k} y^{k-1}, 0\right)$ are replaced by the single element $\left(x^{k-1} y^{k},-x^{k} y^{k-1}, 0\right)$.

For any $\mathbf{u}_{h}=\left(u_{1}, u_{2}, u_{3}\right) \in \mathbf{V}_{h}(K)$, the degrees of freedom are given on face $f$ with unit normal $\mathbf{n}$ and in the interior of $K$ as follows:

$$
\int_{f_{H}} \mathbf{u}_{h} \cdot \mathbf{n} q \mathrm{~d} A, \quad \forall q \in Q_{k, k}\left(f_{H}\right) \backslash\left\{x^{k} y^{k}\right\}, \text { for each horizontal face } f_{H},
$$

$\int_{f_{V}} \mathbf{u}_{h} \cdot \mathbf{n} q \mathrm{~d} A, \quad \forall q \in Q_{k, k}\left(f_{V}\right)$, for each vertical face $f_{V}$,

$\int_{K} u_{3} \phi_{3} \mathrm{~d} \mathbf{x}, \quad \forall \phi_{3} \in Q_{k, k, k-1}^{*} \oplus \operatorname{span}\left\{x^{k+1} y^{i}, x^{i} y^{k+1}, i=0, \ldots, k-1\right\}$,

$$
\int_{K}\left(u_{1} \phi_{1}+u_{2} \phi_{2}\right) \mathrm{d} \mathbf{x}, \quad \forall\left(\phi_{1}, \phi_{2}, 0\right) \in \Psi_{h}(K) .
$$

Then, the number of conditions is $2\left\{(k+1)^{2}\right.$ $-1\}+4(k+1)^{2}+\left\{k(k+1)^{2}-(k-1)+2 k\right\}+2\left\{k(k+1)^{2}-\right.$ $k\}-1$. We start our analysis of this element by showing that the element is unisolvent.
Theorem 1. A vector function $\mathbf{u}_{h}=\left(u_{1}, u_{2}, u_{3}\right) \in \mathbf{V}_{h}(K)$ is uniquely determined by the degrees of freedom (14)-(17).

Proof. We first note that the dimension of $\mathbf{V}_{h}(K)$ is $3 k^{3}+12 k^{2}+14 k+4$, and this is also the number of degrees of freedom. Hence, it suffices to prove that if all the conditions are zero, then $\mathbf{u}_{h}=0$. Since $\mathbf{u}_{h} \cdot \mathbf{n} \in Q_{k, k}\left(f_{V}\right)$ on the vertical faces, (15) implies $\mathbf{u}_{h} \cdot \mathbf{n}=0$ for each vertical face. Then, we have

$$
\begin{aligned}
& u_{1}=x(1-x) v_{1}, \\
& u_{2}=y(1-y) v_{2}, \\
& \mathbf{v}=\left(v_{1}, v_{2}, 0\right) \in \Psi_{h}(K) .
\end{aligned}
$$

Choosing $\phi=\mathbf{v}$ in (17) shows that $\mathbf{v}=0$. Hence, $u_{1}=u_{2}=0$.

Now, consider $u_{3} \in Q_{k, k, k+1}^{*}(K) \oplus \operatorname{span}\left\{x^{k+1} y^{i}, x^{i} y^{k+1}\right.$, $i=0, \ldots, k-1\}$. We note that

$$
u_{3}=s+r_{1} z^{k}+r_{2} z^{k+1}
$$

for some $s \in Q_{k, k, k-1}^{*}(K) \oplus \operatorname{span}\left\{x^{k+1} y^{i}, x^{i} y^{k+1}, i=0, \ldots, k-1\right\}$ and $r_{1}, r_{2} \in Q_{k, k}\left(f_{H}\right) \backslash\left\{x^{k} y^{k}\right\}$. Then, the degrees of freedom (16) implies

$$
u_{3}=r_{1}^{\prime} z^{k}+r_{2}^{\prime} z^{k+1}, \quad \forall r_{1}^{\prime}, r_{2}^{\prime} \in Q_{k, k}\left(f_{H}\right) \backslash\left\{x^{k} y^{k}\right\} .
$$

From (14), we prove that $u_{3}=0$, and we are done.

Definition 2. For the scalar variable, we define 


$$
W_{h}(K)=Q_{k, k, k}^{*} \backslash\left\{x^{k} y^{k}\right\}
$$

The dimension of $W_{h}(K)$ is $\left\{(k+1)^{3}-k\right\}-1=$ $k^{3}+3 k^{2}+2 k$. Also, we know that

$$
\begin{aligned}
& \text { (1) } \operatorname{dim} \mathbf{V}_{h}(K)=W_{h}(K), \\
& \text { (2) } W_{h} \subset W
\end{aligned}
$$

Since $\mathbf{V}_{h} \nsubseteq \mathbf{V}$, we cannot guarantee that the bilinear forms $a(\cdot, \cdot)$ and $b(\cdot, \cdot)$ make sense for functions of $\mathbf{V}_{h}$. So, we define extensions to the larger space $\mathbf{X}_{h}=\mathbf{V}_{h}+H(\operatorname{div}, \Omega)$. Let

$$
\begin{aligned}
& a_{h}(\mathbf{u}, \mathbf{v})=\sum_{i} \int_{K_{i}} \kappa^{-1} \mathbf{u v d} \mathbf{x}, \quad \forall \mathbf{u}, \mathbf{v} \in \mathbf{X}_{h}, \\
& b_{h}(\mathbf{v}, p)=-\sum_{i} \int_{K_{i}} p \operatorname{div} \mathbf{v} \mathrm{d} \mathbf{x}, \quad \forall p \in W_{h}, \forall \mathbf{v} \in \mathbf{X}_{h} .
\end{aligned}
$$

We equip $\mathbf{X}_{h}$ with a norm which is an extension of $\|\cdot\|_{H(\operatorname{div}, \Omega)}$ :

$$
\|\mathbf{u}\|_{\mathbf{X}_{h}}^{2}=\sum_{i}\|\mathbf{u}\|_{H\left(\operatorname{div}, K_{i}\right)}^{2}, \quad \forall \mathbf{u} \in \mathbf{X}_{h}
$$

For $W_{h}$, we equip $L^{2}$-norm. Now, we have the MFE problem corresponding to (6): find $\left(\mathbf{u}_{h}, p_{h}\right) \in \mathbf{X}_{h} \times W_{h}$ such that

$$
\begin{aligned}
a_{h}\left(\mathbf{u}_{h}, \mathbf{v}_{h}\right)+b_{h}\left(\mathbf{v}_{h}, p_{h}\right) & =0, \quad \forall \mathbf{v}_{h} \in \mathbf{V}_{h}, \\
b_{h}\left(\mathbf{u}_{h}, q_{h}\right) & =-\left(f, q_{h}\right), \quad \forall q_{h} \in W_{h} .
\end{aligned}
$$

It is easy to show that the bilinear forms $a_{h}(\cdot, \cdot)$ and $b_{h}(\cdot, \cdot)$ satisfy the following estimate:

$$
\begin{aligned}
& a_{h}\left(\mathbf{u}_{h}, \mathbf{v}_{h}\right) \leq c_{1}\left\|\mathbf{u}_{h}\right\| \mathbf{x}_{h}\left\|\mathbf{v}_{h}\right\| \mathbf{x}_{h}, \\
& b_{h}\left(\mathbf{v}_{h}, p_{h}\right) \leq c_{2}\left\|p_{h}\right\| W_{h}\left\|\mathbf{v}_{h}\right\| \mathbf{x}_{h},
\end{aligned}
$$

for some positive constants $c_{1}$ and $c_{2}$.

Given a typical box element $K$, its boundary $\partial K$ consists of six planar pieces which we distinguish into two kinds: the horizontal plane by $f_{H}$ and the vertical plane $f_{V}$. We define an interpolation operator $\boldsymbol{\Pi}_{h}: \mathbf{H}^{k+1}(K) \longrightarrow \mathbf{V}_{h}(K)$ by

$$
\begin{aligned}
& \int_{f_{H}}\left(\mathbf{u}-\Pi_{h} \mathbf{u}\right) \cdot \mathbf{n} q \mathrm{~d} A=0, \quad \forall q \in Q_{k, k}\left(f_{H}\right) \backslash\left\{x^{k} y^{k}\right\} \\
& \int_{f_{V}}\left(\mathbf{u}-\Pi_{h} \mathbf{u}\right) \cdot \mathbf{n} q \mathrm{~d} A=0, \quad \forall q \in Q_{k, k}\left(f_{V}\right) \\
& \int_{K}\left(u_{3}-\Pi_{h} u_{3}\right) \phi_{3} \mathrm{~d} \mathbf{x}=0, \quad \forall \phi_{3} \in Q_{k, k, k-1}^{*} \oplus \operatorname{span}\left\{x^{k+1} y^{i}, x^{i} y^{k+1}, i=0, \ldots, k-1\right\}, \\
& \int_{K}\left\{\left(u_{1}-\Pi_{h} u_{1}\right) \phi_{1}+\left(u_{2}-\Pi_{h} u_{2}\right) \phi_{2}\right\} \mathrm{d} \mathbf{x}=0, \quad \forall\left(\phi_{1}, \phi_{2}, 0\right) \in \Psi_{h}(K) .
\end{aligned}
$$

Then, we have the following lemma.

Lemma 1. If $\boldsymbol{\Pi}_{h} \mathbf{u}$ is the interpolation of $\mathbf{u}$, then we have

$$
\int_{K} \operatorname{div}\left(\mathbf{u}-\Pi_{h} \mathbf{u}\right) q \mathrm{~d} \mathbf{x}=0, \quad \forall \mathbf{u} \in \mathbf{V}_{h}(K), q \in W_{h}(K) .
$$

Proof. First, let $q \in W_{h}(K)$. Then, we know that $\left.q\right|_{f_{H}} \in Q_{k, k}\left(f_{H}\right) \backslash\left\{x^{k} y^{k}\right\}$ for each horizontal face $f_{H}$ and $\left.q\right|_{f_{V}} \in Q_{k, k}\left(f_{V}\right)$ for each vertical face $f_{V}$. Also, we see that

$$
\begin{aligned}
& \partial_{x} q \in Q_{k-1, k, k}^{*} \backslash\left\{x^{k-1} y^{k}\right\}, \\
& \partial_{y} q \in Q_{k, k-1, k}^{*} \backslash\left\{x^{k} y^{k-1}\right\}, \\
& \partial_{z} q \in Q_{k, k, k-1}^{*} \backslash\left\{x^{k} y^{k}\right\} .
\end{aligned}
$$

Since $\left(\partial_{x} q, \partial_{y} q, 0\right) \in \Psi_{k}(K) \quad$ and $\quad \partial_{z} q \in Q_{k, k, k-1}^{*} \oplus$ $\left\{x^{k+1} y^{i}, x^{i} y^{k+1}, i=0, \ldots, k-1\right\}$, by the definition of $\boldsymbol{\Pi}_{h}$ and that $q \in W_{h}(K)$ and by (29)-(31), we have

$$
\begin{aligned}
\int_{K}( & \left.\operatorname{div} \Pi_{h} \mathbf{u}\right) q \mathrm{~d} \mathbf{x}=\int_{\partial K} \Pi_{h} \mathbf{u} \cdot \mathbf{n} q \mathrm{~d} A-\int_{K} \Pi_{h} \mathbf{u} \cdot \nabla q \mathrm{~d} \mathbf{x} \\
= & \left\{\int_{\partial f_{H}} \boldsymbol{\Pi}_{h} \mathbf{u} \cdot \mathbf{n} q \mathrm{~d} A+\int_{\partial f_{V}} \boldsymbol{\Pi}_{h} \mathbf{u} \cdot \mathbf{n} q \mathrm{~d} A\right\} \\
& -\left\{\int_{K} \boldsymbol{\Pi}_{h} u_{3} \partial_{z} q \mathrm{~d} \mathbf{x}+\int_{K}\left(\boldsymbol{\Pi}_{h} u_{1} \partial_{x} q+\Pi_{h} u_{2} \partial_{y} q\right) \mathrm{d} \mathbf{x}\right\} \\
= & \left\{\int_{\partial f_{H}} \mathbf{u} \cdot \mathbf{n} q \mathrm{~d} A+\int_{\partial f_{V}} \mathbf{u} \cdot \mathbf{n} q \mathrm{~d} A\right\} \\
& -\left\{\int_{K} u_{3} \partial_{z} q \mathrm{~d} \mathbf{x}+\int_{K}\left(u_{1} \partial_{x} q+u_{2} \partial_{y} q\right) \mathrm{d} \mathbf{x}\right\} \\
= & \int_{\partial K} \mathbf{u} \cdot \mathbf{n} q \mathrm{~d} A-\int_{K} \mathbf{u} \cdot \nabla q \mathrm{~d} \mathbf{x} \\
= & \int_{K} \operatorname{div} \mathbf{u} q \mathrm{~d} \mathbf{x} .
\end{aligned}
$$

Let $B$ be an operator from $X_{h}$ to $W^{\prime}$ defined by $B(\mathbf{v}, q)=b(\mathbf{v}, q)$, for all $\mathbf{v} \in X_{h}$ and $q \in W$. Similarly, we define $B_{h}: \mathbf{V}_{h} \longrightarrow W_{h}^{\prime}$ by $B_{h}\left(\mathbf{v}_{h}, q_{h}\right)=b_{h}\left(\mathbf{v}, q_{h}\right)$ for all $\mathbf{v}_{h} \in \mathbf{V}_{h}$ and $q_{h} \in W_{h}$.

To confirm the stability of discrete problem (25), we let 


$$
\begin{aligned}
N\left(B_{h}\right) & =\left\{\mathbf{v}_{h} \in \mathbf{V}_{h} \mid b_{h}\left(\mathbf{v}_{h}, q_{h}\right)=0, \quad \forall q_{h} \in W_{h}\right\}, \\
N(B) & =\left\{\mathbf{v} \in \mathbf{X}_{h}|\operatorname{divv}|_{K}=0, \quad \forall K \in \mathscr{T}_{h}\right\}, \\
N\left(B_{h}^{*}\right) & =\left\{p_{h} \in W_{h} \mid b_{h}\left(\mathbf{v}_{h}, p_{h}\right)=0, \quad \forall \mathbf{v}_{h} \in \mathbf{V}_{h}\right\} .
\end{aligned}
$$

By the definitions and the fact $\operatorname{div} \mathbf{V}_{h}=W_{h}$, we see that $N\left(B_{h}\right) \subset N(B)$, which leads to the following lemma.

Lemma 2. We have

$$
\sup _{\mathbf{v}_{h} \in N\left(B_{h}\right)} \frac{a_{h}\left(\mathbf{v}_{h}, \mathbf{w}_{h}\right)}{\left\|\mathbf{v}_{h}\right\|_{\mathbf{X}_{h}}} \geq \alpha\left\|\mathbf{w}_{h}\right\|_{\mathbf{x}_{h}}, \quad \forall \mathbf{w}_{h} \in N\left(B_{h}\right),
$$

for some positive constant $\alpha$ independent of $h$.

By Lemma 1, the following inf-sup condition can be shown by standard technique [12].

Lemma 3. We have

$$
\sup _{\mathbf{v}_{h} \in \mathbf{X}_{h}} \frac{b_{h}\left(\mathbf{v}_{h}, q_{h}\right)}{\left\|\mathbf{v}_{h}\right\|_{\mathbf{X}_{h}}} \geq \beta\left\|q_{h}\right\|_{W_{h} / N\left(B_{h}^{*}\right)}, \quad \forall q_{h} \in W_{h} .
$$

Theorem 2. Problem (25) has a unique solution.

Proof. The result follows from Lemmas 2 and 3 and Babuska-Brezzi theory.

\section{Error Estimates}

To prove convergence of our method, we need to estimate the approximation error and the consistency error. Since $\mathbf{V}_{h}$ and $W_{h}$ have polynomials of degree $k$, we can easily obtain the following approximation error of order $k$ :

$$
\inf _{\mathbf{v}_{h} \in \mathbf{V}_{h}}\left\|\mathbf{u}-\mathbf{v}_{h}\right\|_{\mathbf{v}_{h}}+\inf _{q_{h} \in W_{h}}\left\|p-q_{h}\right\|_{W_{h}} \leq C h^{k+1}\|p\|_{H^{k+2}(\Omega)}
$$

However, to prove the consistency error is more difficult. We postpone it until later.

To obtain error estimate, we need the following theorem which is essentially given in [10], but we include it for the completeness.

Theorem 3. Let $(\mathbf{u}, p) \in \mathbf{V} \times W$ be the solution of (6) and $\left(\mathbf{u}_{h}, p_{h}\right) \in \mathbf{V}_{h} \times W_{h}$ be the discrete solution of (25). Then, we obtain the following error estimates:

$$
\begin{aligned}
& \left\|\mathbf{u}-\mathbf{u}_{h}\right\|_{\mathbf{X}_{h}}+\left\|p-p_{h}\right\| \theta_{W_{h}} \\
& \leq C\left(\inf _{\mathbf{v}_{h} \in \mathbf{V}_{h}}\left\|\mathbf{u}-\mathbf{v}_{h}\right\|_{\mathbf{V}_{h}}+\inf _{q_{h} \in W_{h}}\left\|p-q_{h}\right\|_{W_{h}}\right. \\
& \left.\quad+\sup _{\mathbf{v}_{h} \in \mathbf{V}_{h}} \frac{\left|a_{h}\left(\mathbf{u}, \mathbf{v}_{h}\right)+b_{h}\left(\mathbf{v}_{h}, p\right)\right|}{\left\|\mathbf{v}_{h}\right\|_{\mathbf{x}_{h}}}\right) .
\end{aligned}
$$

Proof. For an arbitrary $\mathbf{v}_{h} \in \mathbf{V}_{h}$, we let $\mathbf{w}_{h}=\Pi_{h}\left(\mathbf{u}-\mathbf{v}_{h}\right)$ so that

$$
b_{h}\left(\mathbf{w}_{h}, q_{h}\right)=b_{h}\left(\mathbf{u}-\mathbf{v}_{h}, q_{h}\right), \quad \forall q_{h} \in W_{h} .
$$

Then, $\mathbf{x}_{h}=\mathbf{v}_{h}+\mathbf{w}_{h}$ satisfies

$$
b_{h}\left(\mathbf{x}_{h}, q_{h}\right)=b_{h}\left(\mathbf{u}, q_{h}\right), \quad \forall q_{h} \in W_{h} .
$$

Hence, $\mathbf{u}_{h}-\mathbf{x}_{h} \in N\left(B_{h}\right)$, and we have the following inequality from the coercivity (Lemma 2) of $a_{h}(\cdot, \cdot)$ on $N\left(B_{h}\right)$ and (25):

$$
\begin{aligned}
\alpha\left\|\mathbf{u}_{h}-\mathbf{x}_{h}\right\|_{\mathbf{X}_{h}} \leq & \sup _{\mathbf{v}_{h} \in N\left(B_{h}\right)} \frac{1}{\left\|\mathbf{v}_{h}\right\|_{\mathbf{X}_{h}}}\left|a_{h}\left(\mathbf{v}_{h}, \mathbf{u}_{h}-\mathbf{x}_{h}\right)\right| \\
& \leq \sup _{\mathbf{v}_{h} \in N\left(B_{h}\right)} \frac{1}{\left\|\mathbf{v}_{h}\right\|_{\mathbf{X}_{h}}}\left|a_{h}\left(\mathbf{v}_{h}, \mathbf{u}_{h}-\mathbf{u}\right)+a_{h}\left(\mathbf{v}_{h}, \mathbf{u}-\mathbf{x}_{h}\right)\right| \\
& \leq \sup _{\mathbf{v}_{h} \in N\left(B_{h}\right)} \frac{1}{\left\|\mathbf{v}_{h}\right\|_{\mathbf{X}_{h}}} \mid a_{h}\left(\mathbf{v}_{h}, \mathbf{u}-\mathbf{x}_{h}\right)-b_{h}\left(\mathbf{v}_{h}, q_{h}-p\right) \\
& -\left\{a_{h}\left(\mathbf{u}, \mathbf{v}_{h}\right)+b_{h}\left(\mathbf{v}_{h}, p\right)\right\} \mid,
\end{aligned}
$$


for any $q_{h} \in W_{h}$. We know that both bilinear forms are bounded. From this, we get

$$
\begin{aligned}
\alpha\left\|\mathbf{u}_{h}-\mathbf{x}_{h}\right\|_{X} \leq c_{1}\left\|\mathbf{u}-\mathbf{x}_{h}\right\|_{X}+c_{2}\left\|p-q_{h}\right\|_{W} \\
+\sup _{\mathbf{v}_{h} \in N\left(B_{h}\right)} \frac{\left|a_{h}\left(\mathbf{u}, \mathbf{v}_{h}\right)+b_{h}\left(\mathbf{v}_{h}, p\right)\right|}{\left\|\mathbf{v}_{h}\right\|_{X}}, \\
\alpha\left\|\mathbf{u}-\mathbf{u}_{h}\right\|_{\mathbf{X}_{h}} \leq \alpha\left(\left\|\mathbf{u}-\mathbf{x}_{h}\right\|_{\mathbf{X}_{h}}+\left\|\mathbf{u}_{h}-\mathbf{x}_{h}\right\|_{\mathbf{X}_{h}}\right) \\
=\left(\alpha+c_{1}\right)\left\|\mathbf{u}-\mathbf{x}_{h}\right\|_{X}+c_{2}\left\|p-q_{h}\right\|_{W} \\
+\sup _{\mathbf{v}_{h} \in N\left(B_{h}\right)} \frac{\left|a_{h}\left(\mathbf{u}, \mathbf{v}_{h}\right)+b_{h}\left(\mathbf{v}_{h}, p\right)\right|}{\left\|\mathbf{v}_{h}\right\|_{X}} .
\end{aligned}
$$

Therefore,

$$
\begin{aligned}
\left\|\mathbf{u}-\mathbf{u}_{h}\right\|_{\mathbf{X}_{h}} \leq & \left(1+\frac{c_{1}}{\alpha}\right)\left\|\mathbf{u}-\mathbf{x}_{h}\right\|_{\mathbf{X}_{h}}+\frac{c_{2}}{\alpha}\left\|p-q_{h}\right\|_{\mathbf{X}_{h}} \\
& +\frac{1}{\alpha} \sup _{\mathbf{v}_{h} \in N\left(B_{h}\right)} \frac{\left|a_{h}\left(\mathbf{u}, \mathbf{v}_{h}\right)+b_{h}\left(\mathbf{v}_{h}, p\right)\right|}{\left\|\mathbf{v}_{h}\right\|_{\mathbf{X}_{h}}} .
\end{aligned}
$$

From (35) and (38), we also have

$\left\|\mathbf{u}-\mathbf{x}_{h}\right\|_{\mathbf{X}_{h}} \leq\left\|\mathbf{u}-\mathbf{v}_{h}\right\|_{\mathbf{X}_{h}}+\left\|\mathbf{w}_{h}\right\|_{\mathbf{X}_{h}} \leq\left(1+\frac{c_{2}}{\beta}\right)\left\|\mathbf{u}-\mathbf{v}_{h}\right\|_{\mathbf{X}_{h}}$.

By (42) and (43), we have the following estimate:

$$
\begin{aligned}
\left\|\mathbf{u}-\mathbf{u}_{h}\right\|_{\mathbf{X}_{h}} \leq & \left(1+\frac{c_{1}}{\alpha}\right)\left(1+\frac{c_{2}}{\beta}\right)\left\|\mathbf{u}-\mathbf{v}_{h}\right\|_{\mathbf{X}_{h}}+\frac{c_{2}}{\alpha}\left\|p-q_{h}\right\|_{\mathbf{X}_{h}} \\
& +\frac{1}{\alpha} \sup _{\mathbf{v}_{h} \in N\left(B_{h}\right)} \frac{\left|a_{h}\left(\mathbf{u}, \mathbf{v}_{h}\right)+b_{h}\left(\mathbf{v}_{h}, p\right)\right|}{\left\|\mathbf{v}_{h}\right\|_{\mathbf{X}_{h}}} .
\end{aligned}
$$

Using the first equation of (25), we know that $a_{h}\left(\mathbf{u}-\mathbf{u}_{h}, \mathbf{v}_{h}\right)+b_{h}\left(\mathbf{v}_{h}, p-p_{h}\right)=a_{h}\left(\mathbf{u}, \mathbf{v}_{h}\right)+b_{h}\left(\mathbf{v}_{h}, p\right), \quad \forall \mathbf{v}_{h} \in \mathbf{V}_{h}$.

Then, $b_{h}\left(\mathbf{v}_{h}, p-p_{h}\right)=-a\left(\mathbf{u}-\mathbf{u}_{h}, \mathbf{v}_{h}\right)+a_{h}\left(\mathbf{u}, \mathbf{v}_{h}\right)+b_{h}\left(\mathbf{v}_{h}, p\right), \quad \forall \mathbf{v}_{h} \in \mathbf{V}_{h}$.

From (35), we obtain

$$
\left\|p-p_{h}\right\|_{W_{h} / N\left(B_{h}^{*}\right)} \leq \frac{1}{\beta} \sup _{\mathbf{v}_{h} \in \mathbf{V}_{h}} \frac{\left|b_{h}\left(\mathbf{v}_{h}, p-p_{h}\right)\right|}{\left\|\mathbf{v}_{h}\right\|_{\mathbf{X}_{h}}} \leq \frac{1}{\beta}\left(c_{3}\left\|\mathbf{u}-\mathbf{u}_{h}\right\|_{\mathbf{X}_{h}}+\sup _{\mathbf{v}_{h} \in \mathbf{V}_{h}} \frac{\left|a_{h}\left(\mathbf{u}, \mathbf{v}_{h}\right)+b_{h}\left(\mathbf{v}_{h}, p\right)\right|}{\left\|\mathbf{v}_{h}\right\|_{\mathbf{X}_{h}}}\right) .
$$

Joining estimates (44) and (47), we obtain

$$
\left\|\mathbf{u}-\mathbf{u}_{h}\right\|_{\mathbf{X}_{h}}+\left\|p-p_{h}\right\|_{W_{h}} \leq C\left(\inf _{\mathbf{v}_{h} \in \mathbf{V}_{h}}\left\|\mathbf{u}-\mathbf{v}_{h}\right\|_{\mathbf{v}_{h}}+\inf _{q_{h} \in W_{h}}\left\|p-q_{h}\right\|_{W_{h}}+\sup _{\mathbf{v}_{h} \in \mathbf{V}_{h}} \frac{\left|a_{h}\left(\mathbf{u}, \mathbf{v}_{h}\right)+b_{h}\left(p, \mathbf{v}_{h}\right)\right|}{\left\|\mathbf{v}_{h}\right\|_{\mathbf{X}_{h}}}\right),
$$

where $C$ is a constant depending only on $c_{1}, c_{2}, c_{3}, \alpha$, and $\beta$.

Now, we state an assumption regarding the consistency errors:

$$
\begin{aligned}
& \text { (A.1) } \sup _{\mathbf{v}_{h} \in \mathbf{V}_{h}} \frac{\left|a_{h}\left(\mathbf{u}, \mathbf{v}_{h}\right)+b_{h}\left(\mathbf{v}_{h}, p\right)\right|}{\left\|\mathbf{v}_{h}\right\|_{\mathbf{X}_{h}}} \\
& \leq C h^{\ell}\|p\|_{H^{k+2}(\Omega)}, \quad \ell=k \text { or } \ell=k+1 .
\end{aligned}
$$

We prove the error estimates.

Theorem 4. Suppose the following regularity holds: the solution ( $\mathbf{u}, p)$ of (6) satisfies $\mathbf{u} \in \mathbf{H}^{k+1}(\Omega), p \in H^{k+2}(\Omega)$, and there exists some constant $C>0$ such that

$$
\|\mathbf{u}\|_{k+1}+\|p\|_{k+2} \leq C\|f\|_{H^{k}(\Omega)} .
$$

If (A.1) is satisfied, then the following estimates hold:

$$
\begin{aligned}
& \left\|\mathbf{u}-\mathbf{u}_{h}\right\|_{\mathbf{X}_{h}}+\left\|p-p_{h}\right\|_{W_{h}} \leq C h^{\min \{k+1, \ell\}}\|p\|_{H^{k+2}}(\Omega) \\
& \quad \leq C h^{\min \{k+1, \ell\}}\|f\|_{H^{k}}(\Omega) .
\end{aligned}
$$

Proof. This follows directly from Theorem 3, Assumption (A.1), and (36).

Now, we prove (A.1) for the case $\ell=k$. The case $\ell=k+1$ is left open.

Proof of (A.1). By the integration by parts, we see that

$$
\begin{aligned}
a_{h}\left(\mathbf{u}, \mathbf{v}_{h}\right)+b_{h}\left(p, \mathbf{v}_{h}\right) & =\sum_{K \in \mathscr{T}_{h}} \int_{K} \kappa^{-1} \mathbf{u v}_{h} \mathrm{~d} \mathbf{x}-\sum_{K \in \mathscr{T}_{h}} \int_{K} p \operatorname{div} \mathbf{v}_{h} \mathrm{~d} \mathbf{x} \\
& =\sum_{K \in \mathscr{T}_{h}} \int_{K} \kappa^{-1} \mathbf{u v}_{h} \mathrm{~d} \mathbf{x}-\sum_{K \in \mathscr{T}_{h}} \int_{\partial K} p \mathbf{v}_{h} \cdot \mathbf{n} \mathrm{d} \sigma+\sum_{K \in \mathscr{T}_{h}} \int_{K} \nabla p \cdot \mathbf{v}_{h} \mathrm{~d} \mathbf{x} \\
& =-\sum_{K \in \mathscr{T}_{h}} \int_{\partial K} p \mathbf{v}_{h} \cdot \mathbf{n} \mathrm{d} \sigma=-\sum_{K \in \mathscr{T}_{h}} \int_{K} \operatorname{div}\left(p \mathbf{v}_{h}\right) \mathrm{d} \mathbf{x} .
\end{aligned}
$$


Let $\mathbb{1}_{h} p$ be a continuous interpolation of $p$ such that $\left.\mathbb{v}_{h} p\right|_{f_{V}} \in Q_{k, k}$ and $\left.\widehat{K}_{h} p\right|_{f_{H}} \in Q_{k, k} \backslash\left\{x^{k} y^{k}\right\}$. Then, by the patch condition (H2(a), H2(b)), we see

$$
\begin{aligned}
\sum_{K \in \mathscr{T}_{h}} \int_{K} \operatorname{div}\left(p \mathbf{v}_{h}\right) \mathrm{d} \mathbf{x}= & \sum_{K \in \mathscr{T}_{h}} \int_{\partial K} p \mathbf{v}_{h} \cdot \mathbf{n} \mathrm{d} \sigma \\
= & \sum_{K \in \mathscr{T}_{h}} \int_{\partial K}\left(p-\mathbb{I}_{h} p\right) \mathbf{v}_{h} \cdot \mathbf{n} \mathrm{d} \sigma \\
= & \sum_{K \in \mathscr{T}_{h}} \int_{K} \operatorname{div}\left[\left(p-\mathbb{1}_{h} p\right) \mathbf{v}_{h}\right] \mathrm{d} \mathbf{x} \\
= & \sum_{K \in \mathscr{T}_{h}} \int_{K} \nabla\left(p-\mathbb{l}_{h} p\right) \cdot \mathbf{v}_{h} \mathrm{~d} \mathbf{x} \\
& +\sum_{K \in \mathscr{T}_{h}} \int_{K}\left(p-\mathbb{I}_{h} p\right) \operatorname{div} \mathbf{v}_{h} \mathrm{~d} \mathbf{x} \\
= & I+I I .
\end{aligned}
$$

We bound I by the Cauchy-Schwarz inequality and interpolation property:

$$
|\mathrm{I}| \leq\left\|p-\mathbb{1}_{h} p\right\|_{L^{2}(\Omega)}\left\|\mathbf{v}_{h}\right\|_{H(\operatorname{div}, \Omega)} \leq C h^{k+1}\|p\|_{H^{k+2}(\Omega)}\left\|\mathbf{v}_{h}\right\|_{H(\operatorname{div}, \Omega)} .
$$

Similarly, we can bound II as

$$
|\mathrm{II}| \leq\left\|\nabla\left(p-\mathbb{1}_{h} p\right)\right\|_{L^{2}(\Omega)}\left\|\mathbf{v}_{h}\right\|_{L^{2}(\Omega)} \leq C h^{k}\|p\|_{H^{k+2}(\Omega)}\left\|\mathbf{v}_{h}\right\|_{L^{2}(\Omega)} .
$$

Combining inequalities (54) and (55), we have

$$
\left|a_{h}\left(\mathbf{u}, \mathbf{v}_{h}\right)+b_{h}\left(p, \mathbf{v}_{h}\right)\right| \leq C h^{k}\|p\|_{H^{k+2}(\Omega)}\left\|\mathbf{v}_{h}\right\|_{H(\operatorname{div}, \Omega)} .
$$

By dividing $\left\|\mathbf{v}_{h}\right\|_{H(\operatorname{div}, \Omega)}$ and taking supremum over $\mathbf{v}_{h}$, we obtain (A.1).

\section{Numerical Results}

In this section, we report some results of the numerical experiment using our new element for $k=1$. We remark that the optimal convergence rate expected with respective Theorem 4 is

$$
\left\|\mathbf{u}-\mathbf{u}_{h}\right\|_{H(\operatorname{div}, \Omega)}+\left\|p-p_{h}\right\|_{L^{2}(\Omega)} \leq C h^{2},
$$

when $p \in H^{3}(\Omega)$ and $\mathbf{u} \in H^{2}(\Omega)$. We solve problem (2) on the unit cube $\Omega=[0,1]^{3}$. The numerical experiments are carried out on uniform triangulation $\mathscr{T}_{h}$ by cubes having size $h=2^{-m}, m=1,2, \ldots, 5$. We consider two examples where the coefficients $\kappa$ are discontinuous across some interfaces. In each example, the pressure (respectively, velocity) variable belongs to $H^{3}$ (respectively, $H^{2}$ ) on each subregion, and we report $L^{2}$-errors for the pressure variable and $H$ (div)-error for the velocity variable in Tables 1 and 2 . We see that we obtain optimal convergence for both examples.

Example 1. We choose $\kappa=1$ for $0<z<(1 / 2)$ and $\kappa=100$ for $(1 / 2) \leq z<1$. The function
TABLe 1: $L^{2}$-error for pressure and $H$ (div)-error of velocity (Example 1).

\begin{tabular}{llccc}
\hline Level & $\left\|p-p_{h}\right\|_{L^{2}}$ & Order & $\left\|\mathbf{u}-\mathbf{u}_{h}\right\|_{H(\text { div })}$ & Order \\
\hline 1 & $3.652 E-04$ & & $1.649 E-02$ & \\
2 & $1.043 E-04$ & 1.807 & $4.626 E-03$ & 1.834 \\
3 & $2.681 E-05$ & 1.960 & $1.188 E-03$ & 1.961 \\
4 & $6.746 E-06$ & 1.991 & $2.990 E-04$ & 1.990 \\
5 & $1.689 E-06$ & 1.998 & $7.488 E-05$ & 1.998 \\
\hline
\end{tabular}

TABle 2: $L^{2}$-error for pressure and $H$ (div)-error of velocity (Example 2).

\begin{tabular}{llccc}
\hline Level & $\left\|p-p_{h}\right\|_{L^{2}}$ & Order & $\left\|\mathbf{u}-\mathbf{u}_{h}\right\|_{H(\text { div })}$ & Order \\
\hline 1 & $1.324 E-05$ & & $2.608 E-03$ & \\
2 & $5.113 E-06$ & 1.373 & $8.617 E-04$ & 1.597 \\
3 & $1.469 E-06$ & 1.799 & $2.327 E-04$ & 1.889 \\
4 & $3.802 E-07$ & 1.950 & $5.932 E-05$ & 1.972 \\
5 & $9.587 E-08$ & 1.988 & $1.490 E-05$ & 1.993 \\
\hline
\end{tabular}

G. JO AND J. H. KIM

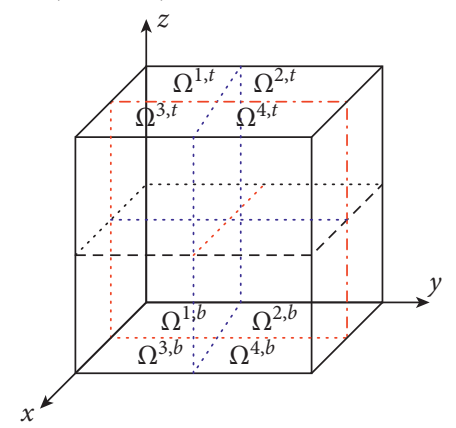

Figure 2: Subregions $\Omega^{i, b}$ and $\Omega^{i, t}, i=1,2,3,4$.

$$
p(x, y, z)=\frac{1}{\kappa} x(1-x) y(1-y) z(z-0.5)(1-z)
$$

is chosen as the exact solution. We report $L^{2}$-errors for the pressure variable and $H$ (div)-error for the velocity variable in Table 1. We observe optimal convergence in both variables.

Example 2. We divide $\Omega=[0,1]^{3}$ by eight octant $\Omega^{i, s}, i=$ $1,2,3,4$ and $s=t, b$ (see Figure 2). Here, $\Omega^{i, b}$ are the four bottom regions $(z \leq 0.5)$ and $\Omega^{i, t}$ are four top regions $(z>0.5)$.

The coefficient $\kappa$ is given by

$$
\begin{aligned}
& \kappa= \begin{cases}1, & \text { for } x \in \Omega^{1, b}, \\
10, & \text { for } x \in \Omega^{2, b}, \\
100, & \text { for } x \in \Omega^{3, b}, \\
1000, & \text { for } x \in \Omega^{4, b}\end{cases} \\
& \kappa= \begin{cases}10, & \text { for } x \in \Omega^{1, t}, \\
100, & \text { for } x \in \Omega^{2, t}, \\
1000, & \text { for } x \in \Omega^{3, t}, \\
1, & \text { for } x \in \Omega^{4, t},\end{cases}
\end{aligned}
$$


and $p=(1 / \kappa) L v$, where $L=(x-0.5)(y-0.5)(z-0.5)$ and $v=x(1-x) y(1-y) z(1-z)$. We report the errors in Table 2. Again, we observe optimal convergence in both variables.

\section{Conclusion}

MFEMs to solve for elliptic equations have been actively studied because approximating the Darcy velocity is as significant as approximating the displacement variable. Most of the MFEMs proposed so far were based on the conforming method in the sense that the space of all vector functions is contained in $H$ (div). On the contrary, the nonconforming approach-based MFEMs were barely studied. In this paper, we suggest a new patch condition under which the nonconforming MFEM has optimal convergence and provides a framework for the convergence. Also, we introduce a new family of nonconforming MFE space which satisfies this condition and provide numerical experiments supporting our analysis.

\section{Data Availability}

The datasets generated and/or analyzed during the current study are available from the corresponding author on reasonable request.

\section{Conflicts of Interest}

The authors declare that they have no conflicts of interest.

\section{Acknowledgments}

The first author was supported by research funds of Kunsan National University. The second author was supported by the National Research Foundation of Korea grant funded by the Ministry of Education, 2017R1C1B5017646. The present work owes much to the advice of Prof. Do. Y. Kwak.

\section{References}

[1] D. Braess, Finite Elements, Cambridge University Press, Cambridge, UK, 2001.

[2] Z. Chen, "Analysis of mixed methods using conforming and nonconforming finite element methods," ESAIM: Mathematical Modelling and Numerical Analysis, vol. 27, no. 1, pp. 9-34, 1993.

[3] P. G. Ciarlet, The Finite Element Method for Elliptic Problems, Vol. 40, SIAM, Philadelphia, PA, USA, 2002.

[4] F. Brezzi, J. Douglas, R. Durán, and M. Fortin, "Mixed finite elements for second order elliptic problems in three variables," Numerische Mathematik, vol. 51, no. 2, pp. 237-250, 1987.

[5] J. C. Nedelec, "Mixed finite elements in $\mathbb{R}^{3}$," Numerische Mathematik, vol. 35, no. 3, pp. 315-341, 1980.

[6] P. A. Raviart and J. M. Thomas, "A mixed finite element method for 2-nd order elliptic problems," Mathematical Aspects of Finite Element Methods, pp. 292-315, Springer, Berlin, Germany, 1977.

[7] W. Xu, D. Liang, and H. Rui, "A multipoint flux mixed finite element method for the compressible Darcy-Forchheimer models," Applied Mathematics and Computation, vol. 315, pp. 259-277, 2017.

[8] C. Srinivas, B. Ramaswamy, and M. F. Wheeler, "Mixed finite element methods for flow through unsaturated porous media," Computational Methods in Water Resources IX, vol. 1, pp. 239-246, 1992.

[9] F. Kikuchi, "Mixed and penalty formulations for finite element analysis of an eigenvalue problem in electromagnetism," Computer Methods in Applied Mechanics and Engineering, vol. 64, no. 1-3, pp. 509-521, 1987.

[10] R. Hiptmair, "Nonconforming mixed discretization of second order elliptic problems," Report TUM M9404, Technische Universitat Munchen, Munich, Germany, 1994.

[11] J. Kim, "A new family of nonconforming mixed finite element methods for second order elliptic problems in $\mathbb{R}^{3}$," International Electronic Journal of Pure and Applied Mathematics, vol. 3, no. 4, pp. 309-320, 2011.

[12] F. Brezzi and M. Fortin, Mixed and Hybrid Finite Element Methods, Springer-Verlag, New York, NY, USA, 1991. 